\section{Gene transfer gets a boost}

Nonviral gene transfer of nondividing cells is notoriously difficult because of inefficient entry of plasmid into the nucleus. In this issue (p. 873), Subramanian et al. develop a new peptide scaffold that overcomes this limitation, yielding a 63 -fold improvement in gene transfer to endothelial cells. Their scaffold combines a nonclassical nuclear localization sequence to improve DNA entry into the nucleus, along with sequences to improve DNA binding.

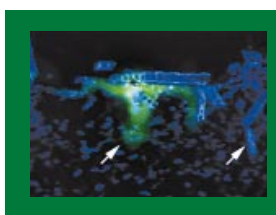

To date, topical gene transfer protocols have employed infectious viral vector physical abrasion, or chemical depilation of the skin. Fan et al. (p. 870) show that specific immune responses to the hepatitis $B$ surface antigen can be induced simply by topical application of naked plasmid DNA in solution to untreated skin. This transfer was shown to depend on the presence of normal hair follicles.

\section{One-step radiolabeling of scFvs}

Radiolabeled single-chain antibody fragments (scFvs) are potentially valuable reagents for imaging and therapeutics. However conventional techniques for binding them to radioisotopes are cumbersome and often reduce their biological activities. On page 897, Plückthun and colleagues describe a new method of formulating technetium-99m, so that it binds conveniently and efficiently to histidine-tagged scFvs. This new chemistry will also make it easier to attach technetium and rhenium to a host of other molecules for therapeutic and diagnostic applications.

\section{Silence is golden}

Ectopic expression of a therapeutic gene can potentially lead to deleterious side effects. On page 865, Mallet and colleagues propose that neuron-restrictive silencer elements (NRESs) may offer advantages over neuronspecific promoters for controlling the expression of such genes in neurons. They use NRESs to limit expression of a reporter gene by an otherwise ubiquitous promoter and demonstrate significant enhancement in the level and control of expression compared with a neuron-specific promoter.

Research Briefs written by Natalie DeWitt, Robert Frederickson, and Andrew Marshall

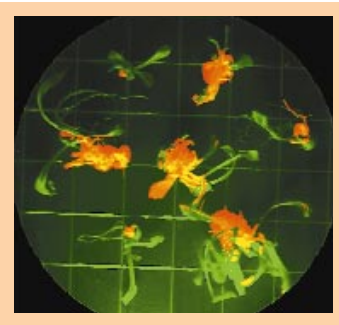

Virtually all genetically engineered crops contain antibiotic resistance genes. This has raised concerns that these genes could be transferred from plants to microbes, with dire consequences to human health. Although the jury is still out on the risks of such horizontal gene transfer (see 832 for Commentary), Chua and colleagues have introduced a new transformation method that avoids the use of antibiotic resistance genes altogether-using instead an inducible cytokinin synthesis gene that stimulates shoot regeneration from transformed cells (p. 916).

\section{Mass attack on proteins}

Genetic strategies to identify DNA-binding proteins often fail to detect interactions dependent upon accessory proteins. Mass spectrometry is a powerful technique to identify the components of macromolecular complexes. Nordhoff and coworkers (p. 884) use this technology to directly identify the components of protein complexes in an extract that bind an immobilized DNA probe.

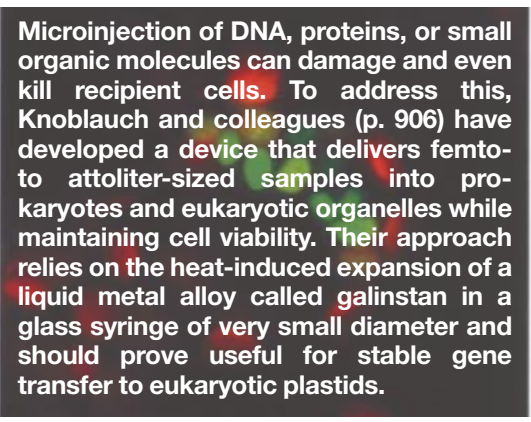

\section{Rationalizing a random approach}

Inteins are proteins that possess both endonuclease and self-splicing activity, allowing self excision and insertion at both the level of the gene and of the protein. Wood and coworkers (p. 889) have used a combination of rational design and an elegant genetic screen in Escherichia coli to engineer a mini-intein that lacks endonuclease activity, and is fine-tuned for use as a tool for recombinant protein purification.

\section{Breeding molecules}

DNA family shuffling is now well established as a powerful technique to tap into and exploit the molecular diversity of gene families. In this issue, Ness and colleagues (p. 893) use this approach to create a library of chimeric subtilisin proteases. Shuffled clones were identified that were improved over the parent enzyme for four distinct enzymatic properties, suggesting the utility of "molecular breeding" to tailor enzymes for diverse functional parameters.

\section{Lighting up transplastomics}

The plastid genomes of plants are prime targets for crop engineering because of their high levels of protein expression and low risk of transgene escape. But a major problem in extending transplastomics to cereal crops has been the difficulty of identifying transformed tissues in the nongreen embryonic cells used for transformation. On page 910 Khan and Maliga describe a way to overcome this bottleneck. They linked a modified form of GFP to an antibiotic resistance marker, making it easy to identify transformed tissues visually.

\section{Human genes find function in yeast}

Scalable and parallel experimental strategies, collectively referred to as "functional genomics," aim to analyze sequence homology, identify protein-protein interactions, and measure gene expression on a genome-wide scale. In this issue, Cismowski and colleagues (p. 906) describe the development of a function-based genomic screen in yeast to identify genes whose products modulate an important mammalian signaling pathway, the Gprotein coupled receptor pathway.

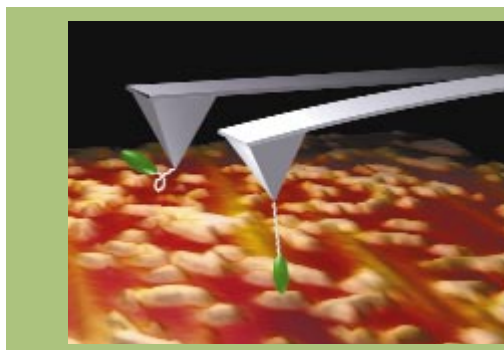

Atomic force microscopy (AFM) is a potentially powerful tool for probing the structure of biological molecules. In this issue (p. 902), Hinterdorfer and colleagues have come up with a new way of imaging antigenic sites on a surface, using an AFM tip with an antibody attached by a flexible tether. The method can be used to simultaneously acquire topographical and molecular recognition images, and so could eventually be used for assigning receptor sites to locations on topographical maps. 